Typeset with jpsj3.cls $<$ ver.1.0 $>$

Full PAPER

\title{
Melting of MgO Studied using a Multicanonical Ensemble Method Combined with a First-Principles Calculation
}

\author{
Yoshihide Yoshimoto * \\ Institute for Solid State Physics, University of Tokyo, 5-1-5 Kashiwa-no-ha, Kashiwa-shi, Chiba, \\ 2778581, Japan
}

\begin{abstract}
Melting of $\mathrm{MgO}$ was studied using a multicanonical ensemble method combined with a first-principles calculation. This approach has been successively performed by using a rather simple functional form for a model inter-atomic potential that is determined from firstprinciples and a novel approximation treating auxiliary degrees of freedom, such as electron thermal excitations, within a multicanonical ensemble method. Although a rather simple model potential was used, this approach could distinguish differences due to the exchangecorrelation potential used in the first-principles calculations. The pressure dependence of the melting point, latent heat, and volume change during melting were studied. The obtained dependence was similar to that reported by Alfè which differs from experimental results. This dependence did not change even with the PBEsol exchange-correlation potential.
\end{abstract}

KEYWORDS: $\mathrm{MgO}$, multicanonical ensemble, first-principles calculation, melting, pressure dependence

\section{Introduction}

Changes in atomic structure during phase transitions has been one of the principal goals in material science. Of the various types of phase transitions, melting, and its reverse process, crystallization, are the most basic. Moreover, these are both technologically important because crystal growth is fundamental to material synthesis as is casting to shape forming.

In another aspect, first-principles calculations has been a powerful theoretical tool in material science because of its ability to treat atomic structures. This ability is one reason why it is expected to contribute future material developments.

In general, material developments can be separated into a cycle of three stages: design, synthesis, and characterization. The synthesis stage is, however, one of the less-developed areas as far as first-principles studies are concerned. Most first-principles studies have contributed to the characterization of materials. With the exception of some advanced work, few have been proposed in the design stage. For this reason, the present study endeavors to treat melting by a first-principles calculation. First-principles treatment of crystal growth and melting, two processes that are strongly related within synthesis work, should become common practice.

In the study of melting employing first-principles calculations, two approaches exist. The

*E-mail address: yosimoto@issp.u-tokyo.ac.jp 
first is the two-phase method, which simulates an interface between two phases to determine melting points $\left(T_{m}\right) .{ }^{1)}$ The other is the thermodynamic integration method $^{2)}$ and related adiabatic switching method. ${ }^{3,4)}$ The first requires a large simulation cell to simulate adequately an interface between two phases. The other does not require such a large simulation cell but requires some reference system whose free energy is well-understood and can be smoothly connected to the target system under study.

The author has previously proposed another first-principles approach to the study of melting, ${ }^{5)}$ based on a combination of the multicanonical ensemble $\operatorname{method}^{6-8)}$ along with a first-principles calculation. It does not require a large simulation cell because it does not simulate an interface. This is important from a first-principles calculation perspective because its computational cost increases rapidly as a function of system size. Furthermore, the approach does not require a common well-understood reference system connecting the free energies of both phases. In general, such systems are not expected to exist.

The present study treats the melting of $\mathrm{MgO}$ by this novel approach. The reason why $\mathrm{MgO}$ was chosen is as follows. (1) Of the four types of crystals, covalent, metallic, ionic, and molecular, $\mathrm{MgO}$ is a typical representative of ionic crystals. (2) $\mathrm{MgO}$ has technological applications. It is a typical refractory material and a typical substrate used in thin film growth. (3) $\mathrm{MgO}$ is one extreme constituent of rocks, which are oxides of $\mathrm{Mg}$, $\mathrm{Si}$, and $\mathrm{Al}$. For this reason, the pressure $(P)$ dependent melting curve of $\mathrm{MgO}$ has been studied extensively in the context of earth science. An experimental study has suggested that $\mathrm{MgO}$ melts at rather low temperature under high pressures. ${ }^{9)}$ (Specifically, Zerr and Boehler observed that MgO melted at $\sim 3500 \mathrm{~K}$ under $\sim 17 \mathrm{GPa}$, and predicted that MgO melts at $\sim 5200 \mathrm{~K}$ under $\sim 135$ GPa.) However, a following first-principles study using the two phase method did not agree with it. ${ }^{10)}$ (i.e., at $4590 \mathrm{~K}$ and $8144 \mathrm{~K}$ under $17 \mathrm{GPa}$ and $135.6 \mathrm{GPa}$, respectively) In addition, another experimental study by Zhang and Fei on ( $\mathrm{Mg}, \mathrm{Fe}) \mathrm{O}$ solidus under high pressure recently claimed higher $T_{m}$ than theoretical values under high pressures from the extrapolation of their result. ${ }^{11)}$ (Even under $7 \mathrm{GPa}$, the estimated $T_{m}$ was $\sim 4500 \mathrm{~K}$. The extrapolated melting point from their result under $17 \mathrm{GPa}$ seems to be $\sim 5500 \mathrm{~K}$.) Altanetively, we can compare the melting slope $\left(d T_{m} / d P\right)$ instead of $T_{m}$ itself, because we can calculate $d T_{m} / d P$ from the Clausius-Clapeyron relation. Recently, Tangeny and Scandolo ${ }^{12)}$ studied this melting slope at $P=0 \mathrm{GPa}$ using a combination of molecular dynamics and density functional calculations. After a detailed discussion, they concluded that the theoretical values are from $\sim 130$ to $\sim 150 \mathrm{~K} / \mathrm{GPa}$, which clearly differs from the ones reporeted by Zerr and Boehler (36 $\mathrm{K} / \mathrm{GPa}$ ). (4) The melting behavior of $\mathrm{MgO}$ is not fully understood. No calorimetric study of latent heat is available for this material. The apparent values ranging from 8 to $30 \mathrm{kcal} \mathrm{mol}^{-1}$ were obtained from binary systems. ${ }^{13)}$

The present paper also reports the application of the PBEsol exchange-correlation (XC) 
potential $^{14,15)}$ to investigate a possible cause for the discrepancy between experimental and theoretical results. This issue may be because of an electronic correlation problem in firstprinciples calculations and the PBEsol claim that it is a better approximation for condensed materials.

The structure of the paper is as follows: First, a brief introduction to the present approach is presented. Second, I address an issue arising with thermal excitation of electrons and its treatment. Third, the definition of an order parameter is discussed within the present approach. Fourth, calculation conditions will be detailed. Fifth, calculational checks are presented. Sixth, comparisons between various XC potentials used in the present approach will be analyzed. Last, the simulated melting behavior at $P=0 \mathrm{GPa}$ and the pressure dependence of melting will be presented for the issues concerning latent heat and melting point curves.

The simulated results will yield values for $T_{m}$, latent heat $(\Delta H)$, and volume change per atom during melting $(\Delta V)$. These properties have significance in material synthesis. For instance, the melting point and its latent heat will be useful in planning crystal growth. The melting point itself becomes important when developing heat-resistant materials. Volume changes during melting will be useful information for casting when precision is important in material shaping. Pressure effects are relevant in high-pressure synthesis work.

\section{Multicanonical ensemble method combined with first-principles calculation}

In this section, a brief review is given highlighting the combination of a multicanonical ensemble method with first-principles calculations.

The multicanonical ensemble method is a type of generalized ensemble method that uses a generalized statistical weight instead of the canonical Boltzmann factor $e^{-\beta E}$. This generalized statistical weight is the estimated inverse of the density of states $\tilde{W}(E)$ for which the probability $P(E)$ of observing some energy $E$ becomes constant. This is because $P(E) \propto W(E) \tilde{W}^{-1}(E) \sim 1$, where, $W(E)$ is the density of states in the system. As a consequence, the energy observed in a molecular dynamics (MD) simulation or a Monte Carlo simulation fluctuates widely so that the tunneling time from one state to another is expected to be short compared with that in a canonical simulation. There, the fluctuation is strongly confined around the expected energy value. $\tilde{W}$ or the estimated entropy $\tilde{S}=k_{B} \log \tilde{W}$ can be generated by the Wang-Landau algorithm. ${ }^{16)}$ This is an "on-the-fly"-type algorithm that is used to obtain $\tilde{S}$ in an efficient manner.

To obtain any physical quantity from a multicanonical simulation, a technique to recompile the simulation run, called re-weighting, ${ }^{17)}$ is used. Unlike in a canonical simulation, a physical quantity at any temperature can be obtained ideally from just a single simulation run by re-weighting.

The multicanonical ensemble method works theoretically for a system with a first-order phase transition. However, naively applying this method to simulate melting of a crystal such 
as silicon or $\mathrm{MgO}$ is still difficult because of the slow tunneling times between the two phases.

To overcome this difficulty, we found in a previous study that the multi-order multithermal $(M O M T)$ ensemble, ${ }^{18)}$ utilizing an order parameter defined using structure factors of the target crystal structure, was useful.5) This ensemble is a two-component multicanonical ensemble. ${ }^{19)}$ Specifically, $\tilde{S}$ becomes a function of both energy and an order parameter. By explicitly treating the crystalline order, we can make the system fluctuate between two phases in short periods.

This extended multicanonical ensemble method can in practice simulate the phase transition with a model inter-atomic potential. Direct application with first-principles calculations is, however, still impractical because the required number of MD steps is far larger than available number $\left(\sim 10^{4}\right)$.

Thermodynamic downfolding is a method that resolves this issue between available number and required number of the steps. This method generates a model inter-atomic potential $U_{M}$ from an accurate inter-atomic potential $U_{A}$ (for instance, one obtained from first-principles) conserving the thermodynamics of the target system as much as possible.

Usually, a model inter-atomic potential is constructed by making it reproduce as much as possible an accurate version on some reference atomic configurations. In thermodynamic downfolding, the reference configurations are a (down-sampled) multicanonical ensemble (simulation run). This choice is thermodynamically meaningful because we can derive any thermal quantity at any temperature by applying a re-weighting over the ensemble. Specifically, a multicanonical ensemble is a representative of the total thermodynamics of a system. Dependence on the chosen atomic configurations is eliminated by making the inter-atomic potential and the configurations self-consistent.

In summary, the combination of the MOMT ensemble method and the thermodynamic downfolding makes multicanonical simulations of melting from first-principles possible. The merits of this approach are as follows: (1) The required simulation cell size is small because we do not simulate an interface between the two phases. (2) We do not have to provide a reference inter-atomic potential for which free energy is well-understood and which can be smoothly connected to the target potential. To provide such a potential is difficult especially for a system with multiple components. (3) Accurate calculations can be performed in parallel because all atomic configurations to be calculated are known from the outset. Therefore, we can utilize inexpensive computer systems with weak interconnects. In addition, this approach does not need any parameter-fitting of some experimental data. Indeed, all parameters in the model inter-atomic potential are determined from first-principles. In this context, this approach represents a type of ab-initio method. The form of the model potential should be regarded as a kind of basis set. In this approach, we can chose it arbitrarily so that the residual in the downfolding becomes small enough. 


\section{Approximation to treat auxiliary degrees of freedom such as thermal excita- tion of electrons}

The rather high melting point of $\mathrm{MgO}$ makes the thermal excitation of electrons have a significant effect on the melting. ${ }^{10)}$ However, in trying to include such effects, the multicanonical ensemble method has a problem treating auxiliary degrees of freedom, such as thermal excitation of electrons to be traced out. In contrast, we can partially trace out the auxiliary degrees of freedom in the canonical ensemble method. This partial trace gives a free energy function as a function of both the principal degrees of freedom (e.g. the atomic positions) and temperature. By using the free energy function instead of the energy function in a Monte-Carlo simulation or a MD simulation, we can deduce a thermal average of a physical quantity that does not directly depend on the auxiliary degrees of freedom. In a multicanonical simulation, however, the energy function itself cannot depend on temperature because the simulation run does not have a physical temperature.

To resolve this issue, an approximation is developed. To understand the approximation, the re-weighting method employed to obtain the average of physical quantity should be reconsidered. This thermal-averaged physical quantity $A$ is calculated by a re-weighting method from the expression

$$
\langle A\rangle=\frac{\sum_{i} A_{i} \tilde{W}\left(E_{i}\right) e^{-\beta E_{i}}}{\sum_{i} \tilde{W}\left(E_{i}\right) e^{-\beta E_{i}}},
$$

where $E_{i}$ is the energy of a configuration $i$ in a multicanonical simulation run.

Here, we note the following fact: the term $\tilde{W}(E) e^{-\beta E}$ has a sharp peak around the expectation value of energy $\bar{E}(\beta)$ (Fig. 1). We can consider this term as a clipping mask for the multicanonical simulation run. Because of this fact, each configuration $i$ has its maxi-

mal contributing temperature $\beta_{i}^{e f f}$, obtained by finding the temperature that maximizes the following expression

$$
\frac{\tilde{W}\left(E_{i}\right) e^{-\beta E}}{\sum_{i} \tilde{W}\left(E_{i}\right) e^{-\beta E_{i}}} .
$$

By using $\beta_{i}^{e f f}$, we can determine a model inter-atomic potential by thermodynamic downfolding as follows: a first-principles calculation is performed at temperature $\beta_{i}^{e f f}$ on each configuration $i$ in the down-sampled multicanonical ensemble. In particular, by using $\beta_{i}^{e f f}$, we can incorporate approximately the temperature dependence of each $i$. The first-principles calculation produces both energy and free energy. For both, thermodynamic downfolding is performed to obtain both the model inter-atomic energy and free energy potentials. The following multicanonical simulation is performed with this inter-atomic free energy potential because the statistical weight is determined by the free energy. To obtain the thermal-averaged energy, however, we have to average the inter-atomic energy potential instead of the free energy 


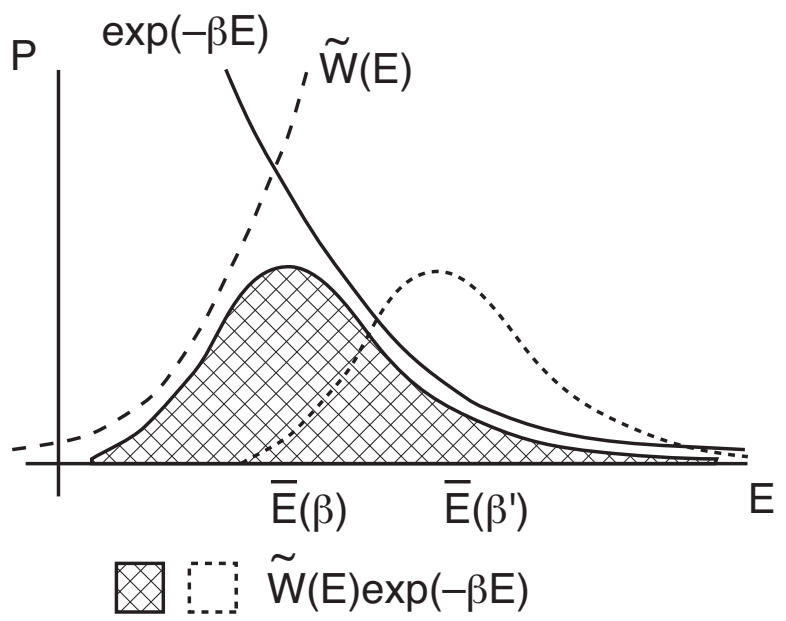

Fig. 1. The term, $\tilde{W}(E) e^{-\beta E}$, for the multicanonical ensemble to obtain physical quantity. $E$ and $P$ are energy and probability, respectively. $\tilde{W}(E)$ is an increasing function, while $e^{-\beta E}$ is a decreasing function. The product, $\tilde{W}(E) e^{-\beta E}$, has a peak at $\bar{E}$ and its position depends on temperature.

potential because in the partial trace formalism we have

$$
\bar{E}(\beta)=\frac{\sum_{i} E_{i} e^{-\beta F_{i}(\beta)}}{\sum_{i} e^{-\beta F_{i}(\beta)}} .
$$

Consequently, in the present approximation, the re-weighting method for energy becomes

$$
\bar{E}(\beta)=\frac{\sum_{i} E_{i} \tilde{W}\left(F_{i}\right) e^{-\beta F_{i}}}{\sum_{i} \tilde{W}\left(F_{i}\right) e^{-\beta F_{i}}} .
$$

In applications to melting, a further improvement is available. We can split the downsampled multicanonical ensemble into crystalline-like and liquid-like configurations by using an order parameter. Therefore, it is reasonable to use the split ensemble including the target configuration $i$ to determine $\beta_{i}^{e f f}$. By this refinement, we can better treat over-heated states and over-cooled states.

\section{The order parameter for multi-order multi-thermal simulation for $\mathrm{MgO}$}

To perform a multi-order multi-thermal simulation efficiently, a rescaled order parameter is introduced:

$O=\sinh \left(\frac{O_{n s}-\frac{1}{2} O_{\max }}{\sqrt{O_{\max } / O_{\alpha}}}\right) / \sinh \left(\frac{1}{2} \sqrt{O_{\alpha} O_{\max }}\right)+\frac{O_{n s}-\frac{1}{2} O_{\max }}{O_{\max }}$, where $O_{n s}=\frac{1}{N_{\mathcal{G}} N_{A}} \sum_{\mathbf{G} \in \mathcal{G}}|s(\mathbf{G})|^{2}$,

where $O_{n s}$ is the non-scaled order parameter adopted in the previous study and $O_{\max }$ and $O_{\alpha}$ are scaling parameters. The set $\mathcal{G}$ is composed of the $N_{\mathcal{G}}$ shortest reciprocal lattice vectors for the crystalline order. $s(\mathbf{G})=\sum_{j} \exp \left(i \mathbf{G} \cdot \mathbf{R}_{j}\right)$ is the structure factor where $\mathbf{R}_{j}$ is the $j$-th atomic position; the sum is over $N_{A}$ atomic positions. For $\mathrm{MgO}$, only the $\mathrm{Mg}$ atom 
J. Phys. Soc. Jpn.

Full PAPER

positions were considered in the calculation of $s(\mathbf{G})$. In the principal calculation condition, which included $32 \mathrm{Mg}$ and $32 \mathrm{O}$ atoms in a cubic cell, $O$ is rescaled within $-3 / 2<O<3 / 2$ with $O_{\max }=32$ and $O_{\alpha}=2$. In addition, $N_{\mathcal{G}}=8$ for this condition.

\section{The calculation conditions}

The functional form of the model inter-atomic potentials $U_{M}$ used in this study was the Born-Huggins-Mayer short-range repulsion potential with a Morse attractive potential, namely

$U_{M}=\sum_{i<j}\left[f_{0} b_{i, j} \exp \left(\frac{a_{i, j}-r_{i, j}}{b_{i, j}}\right)+d_{i, j}\left[\exp \left(-2 \beta_{i, j}\left(r_{i, j}-r_{i, j}^{0}\right)\right)-2 \exp \left(-\beta_{i, j}\left(r_{i, j}-r_{i, j}^{0}\right)\right)\right]\right]$,

where $r_{i, j}$ is the distance between atoms $i$ and $j$. The others, i.e. $a, b, \beta, d$, and $r^{0}$, are parameters that depend on atomic species, and are symmetric with respect to the exchange of their indices. The accurate inter-atomic potential, $U_{A}$, was obtained by a density functional calculation with a plane wave basis set and pseudo-potentials, which was performed by an extended version of the program package TAPP. ${ }^{20}$ ) The majority of the calculations were performed with PBE-type XC potential. ${ }^{21,22)}$ In others, PBEsol-type, ${ }^{14,15)}$ CAPZ-type, ${ }^{23,24)}$ or PW91-type ${ }^{25)} \mathrm{XC}$ potentials were used. The principal cell size, which contained 64 atoms in total, had been used in the previous first-principles study ${ }^{10)}$ to evaluate latent heat and volume change during melting. Only the $\Gamma$ point was sampled in the first Brillouin zone. To keep the effective cut-off energy of the basis set constant against volume change, the method proposed by Bernasconi et al. ${ }^{26)}$ was applied; we have $E_{0}=42.25 \mathrm{Ry}, A=80.0 \mathrm{Ry}$, and $\sigma=0.8 \mathrm{Ry}$. The total number of plane waves in the basis set was 23439 . The effective cut-off $E_{0}=42.25 \mathrm{Ry}$ is rather higher than that in the previous first-principles calculation. ${ }^{10)}$ When 4 irreducible $k$-points were sampled in a downfolding iteration, the changes in the $T_{m}, \Delta H$, and $\Delta V$ compared with the result by $\Gamma$ point sampling were $10 \mathrm{~K}, 2 \mathrm{~kJ} \cdot \mathrm{mol}^{-1}$, and $-0.08 \AA^{3}$, respectively.

The MOMT ensemble MD simulations ${ }^{5)}$ were performed using $^{27-29)}$ an isobaric-isothermal ensemble MD algorithm. ${ }^{30)}$ The reference temperature, $T_{0}$, for MD was 2750, 4350, 5800, and $8000 \mathrm{~K}$ for $P=0,17,47$ and $135.6 \mathrm{GPa}$, respectively. The parameters of the thermostat and the barostat, $\omega_{\zeta}$ and $\omega_{\eta}$ have values $1.0 \times 10^{-3}$ and $5.0 \times 10^{-5}$ in atomic units, respectively. The time step of the simulation was 5 a.u. In the Wang-Landau algorithm that generates the multicanonical weight, a factor, $f$, was added every $100 \mathrm{MD}$ steps and the flatness of its histogram was checked every 30,000 MD steps. The mesh spacings for the partial entropy of the system, ${ }^{5,29)} \delta \tilde{S}=\tilde{S}-U / T_{0}$, actually generated in the algorithm were 0.1 a.u. for $U$ and 0.1 for $O$, respectively. (The unit of the factor and $\delta \tilde{S}$ is $k_{B}$.) The maximum value of $\delta \tilde{S}$ was set at zero. For $P=0 \mathrm{GPa}$ and $P=17 \mathrm{GPa}$, the minimum was kept larger than $-40+2 f$. For $P=47 \mathrm{GPa}$ and $P=135.6 \mathrm{GPa}$, it was kept larger than $-60+2 f$. The same definition was adopted for the flatness of the histogram as employed in the previous 
study. ${ }^{5)}$ The algorithm was continued until a flat histogram was obtained with $f=1 / 128$. At this stage, the multicanonical weight for the production run was obtained. The number of MD steps for the production run was $3 \times 10^{7}$. This was enough to obtain a physically sound smooth temperature dependence for both liquid and crystalline phases by re-weighting.

The procedure for thermodynamic downfolding was as follows.

To begin, the definition of the target function $L$ for downfolding was

$$
L=\sum_{X_{i} \in \mathcal{Q}}\left[\left(\Delta U\left(X_{i}\right)-\langle\Delta U\rangle\right)^{2}+w\left(\left.3 \frac{\partial U_{M}}{\partial V}\right|_{X_{i}} V_{i}-\left.3 \frac{\partial U_{A}}{\partial V}\right|_{X_{i}} V_{i}\right)^{2}\right]
$$

where $\mathcal{Q}$ is a down-sampled multicanonical ensemble. $X_{i}$ and $V_{i}$ represent an atomic configuration and its corresponding cell volume, respectively. $\Delta U=U_{M}-U_{A}$ is the difference between model and accurate inter-atomic potential energy. In addition,

$$
\langle\Delta U\rangle=\frac{1}{|\mathcal{Q}|} \sum_{X_{k} \in \mathcal{Q}} \Delta U\left(X_{k}\right)
$$

Atomic units are employed throughout here. $w=0.02$ is a parameter. Thus, the present definition of $L$ includes a stress term so that the current study can treat pressure dependence.

As in the previous study on silicon, $X_{i}$ was considered in down-sampling for $\mathcal{Q}$ only if

$$
\max _{O} \tilde{S}\left(U\left(X_{i}\right), O\right)-\tilde{S}\left(U\left(X_{i}\right), O\left(X_{i}\right)\right) \leq 7 k_{B}
$$

where $\tilde{S}$ is the estimated entropy by the MOMT ensemble. This is because $X_{i}$ does not otherwise contribute to thermal averages.

At the beginning of the downfolding iteration series, the effect of thermal electronic excitation was not considered. Downfolding was performed only under zero pressure. This stage dealt with generating an initial estimate.

For the first iteration of downfolding, the model inter-atomic potential used was taken from ref. 31. $\left(U_{m}^{0}\right)$ The functional form of this potential comprises the Ewald term plus a Born-Huggins-Mayer term. After the first iteration, it was found that the present form of model potential had the same effectiveness as that with the Ewald term. Because calculations involving the Ewald term are computationally intensive, the subsequent calculations were performed with the present form of the potential.

After the first iteration, two further iterations of downfolding were performed to obtain an initial estimate $\left(U_{m}^{2}\right)$ with which production iterations can proceed. In these iterations, thermal electronic excitation effects were considered.

For the first-principles calculations, the number of sampled configurations in each downfolding is set at 500, while the number of parameters in the present model inter-atomic potential is 9 . In our previous study on silicon which sampled 1000 configurations, ${ }^{5)}$ this number was 16. Therefore, a smaller sampling set was appropriate.

The first production iteration, S1, was performed under zero pressure. This iteration pro- 
duced model potential $U_{M}^{1}$ from $U_{M}^{0}=U_{m}^{2}$.

The second production iteration, S2, was performed under $P=0,17,47$, and 135.6 GPa.

In this iteration, multicanonical simulations were performed with the $U_{M}^{1}$ model potential to generate corresponding down-sampled simulation runs, $\mathcal{Q}^{1}(P)$, for downfolding. From the simulation, physical quantities were also obtained. Using $\mathcal{Q}^{1}(P)$, downfolding was performed to obtain $U_{M}^{2}(P)$ model potentials for each pressure.

The third production iteration, S3, was performed with pressures $P=0,17,47$, and 135.6 GPa. In this iteration, multicanonical simulations for each pressure were performed with the $U_{M}^{2}(P)$ model potentials and physical quantities were obtained from the simulations.

In addition to these main iterations, a branch iteration, $\hat{\mathrm{S}} 3$, with PBEsol was performed with the same pressure settings: $P=0,17,47$, and $135.6 \mathrm{GPa}$. To begin, downfolding was performed from $\mathcal{Q}^{1}(P)$ in S2 to obtain model potentials $\hat{U}_{M}^{3}(P)$. Then, multicanonical simulations were performed using $\hat{U}_{M}^{3}(P)$ to obtain physical quantities. In addition, a further downfolding was performed at $P=0$ to obtain $\hat{U}_{M}^{4}(P=0)$.

Using $\hat{U}_{M}^{4}(P=0)$, a checking iteration, $\hat{\mathrm{S}} 4$, was performed. From a multicanonical simulation, physical quantities were obtained to be used as a comparison against results with $\hat{U}_{M}^{3}(P=0)$.

In addition to PBEsol, another branch iteration, $\tilde{\mathrm{S}} 3$, with CAPZ was performed with $P$ $=0$ GPa. At first, downfolding was performed from $\mathcal{Q}^{1}(P=0)$ in S2 to obtain $\tilde{U}_{M}^{3}(P=0)$ model potentials. Subsequently, multicanonical simulations were performed using $\tilde{U}_{M}^{3}(P=0)$ to generate a down-sampled simulation run, $\tilde{\mathcal{Q}}^{2}(P=0)$. For this branch with CAPZ, the appropriate $T_{0}$ was $3400 \mathrm{~K}$. With $\tilde{\mathcal{Q}}^{2}(P=0)$, a further downfolding was performed to obtain $\tilde{U}_{M}^{4}(P=0)$.

Using $\tilde{U}_{M}^{4}(P=0)$, a production iteration, $\tilde{\mathrm{S}} 4$, was performed. A multicanonical simulation was performed to obtain physical quantities. By comparing physical quantities of this iteration to previous values, it was found that this iteration is sufficient to obtain results that converged.

As for the computation of the above procedure, the required computational cost for one iteration of downfolding and the following MOMT simulation is commented in Appendix D.

\section{Calculational checks}

First, the effect of thermal electronic excitation was checked at $P=0 \mathrm{GPa}$, by producing a downfolded potential starting from an $U_{M}^{0}$ in the absence of the effect. This procedure constituted one iteration of downfolding. Consequently, $U_{M}^{1}$ is the corresponding downfolded potential with the effect. Changes in $T_{m}, \Delta H$ and $\Delta V$ were $150 \mathrm{~K},-6 \mathrm{~kJ} \cdot \mathrm{mol}^{-1}$, and -0.17 $\AA^{3}$, respectively. The thermal electronic excitation clearly decreases the melting point. Because the energy gap in the electronic structure for the liquid state vanishes, the decrease in the free energy by thermal electronic excitation is significant for the liquid state. Therefore, it is natural to obtain a lower melting point when the effect is considered. 
Table I. Simulation cell size dependence of melting under $P=0$ GPa. A cubic cell containing 64 atoms, a fcc cell containing 128 atoms, and a cubic cell containing 216 atoms are compared. $U_{M}^{2}(P=0), \hat{U}_{M}^{3}(P=0)$, and $\tilde{U}_{M}^{4}(P=0)$ model inter-atomic potentials were used for PBE, PBEsol, and CAPZ simulations, respectively. $P, T_{m}, V_{x}, V_{l}, \Delta V, \Delta H$, and $\Delta S$ are pressure, melting point, volume per atom for crystalline state at $T_{m}$, volume per atom for liquid state at $T_{m}$, volume change per atom during melting, latent heat, and entropy change per atom during melting, respectively.

\begin{tabular}{|c|c|c|c|c|c|c|c|}
\hline atom & $\begin{array}{c}T_{m} \\
\mathrm{~K}\end{array}$ & $\begin{array}{l}V_{x} \\
\AA^{3}\end{array}$ & $\begin{array}{l}V_{l} \\
\AA^{3}\end{array}$ & $\begin{array}{l}\Delta V \\
\AA^{3}\end{array}$ & $\begin{array}{c}\Delta H \\
\mathrm{~kJ} \cdot \mathrm{mol}^{-1}\end{array}$ & $\begin{array}{l}\Delta S \\
k_{B}\end{array}$ & \\
\hline 64 & 2975 & 10.93 & 14.44 & 3.52 & 85 & 1.7 & PBE \\
\hline 128 & 2850 & 10.77 & 14.50 & 3.73 & 86 & 1.8 & PBE \\
\hline 216 & 2820 & 10.78 & 14.00 & 3.22 & 82 & 1.7 & PBE \\
\hline 64 & 3230 & 10.66 & 13.67 & 3.01 & 87 & 1.6 & PBEsol \\
\hline 128 & 3120 & 10.57 & 13.80 & 3.23 & 89 & 1.7 & PBEsol \\
\hline 216 & 3060 & 10.52 & 13.26 & 2.74 & 86 & 1.7 & PBEsol \\
\hline 64 & 3460 & 10.43 & 13.26 & 2.84 & 94 & 1.6 & CAPZ \\
\hline 128 & 3340 & 10.31 & 13.28 & 2.96 & 93 & 1.7 & CAPZ \\
\hline 216 & 3270 & 10.29 & 12.82 & 2.53 & 88 & 1.6 & CAPZ \\
\hline
\end{tabular}

Second, the simulation cell-size dependence was studied utilizing two methods.

The first method performs a MOMT simulation with a 128-atom fcc cell and a 216-atom cubic cell using a model potential obtained by the 64 atom cubic cell. The result is shown in Table I. For simulations with larger cells, some techniques were introduced that have been described in detail in the appendix A. The model potential used was $U_{M}^{2}(P=0), \hat{U}_{M}^{3}(P=0)$, and $\tilde{U}_{M}^{4}(P=0)$. From the result, the simulation based on a 64 -atom cubic cell seems to overestimate $T_{m}$ by $150-200 \mathrm{~K}$. The errors in $\Delta V$ and $\Delta H$ were $\sim 0.5 \AA^{3}$ and $\sim 5 \mathrm{~kJ} \cdot \mathrm{mol}^{-1}$, respectively.

The decrease in the melting point is understandable because the larger simulation cell probably enables the liquid state to take more configurations compared with the crystalline state. Therefore, the relative free energy of the liquid state should decrease in a larger simulation cell. This relative decrease in the free energy prompts a lower melting point.

The second method used to perform calculational checks involves generating a downsampled multicanonical ensemble with a model potential for a larger cell and comparing this potential to the accurate potential for the same ensemble. Using a down-sampled simulation run with a 128 atom fcc cell, the $U_{M}^{2}(P=0)$ model potential were compared with the firstprinciples potential. The comparison is shown in Fig. 2. The dashed line in the figure is a guide for the correspondence between the two potentials. In this figure, the model potential and the 


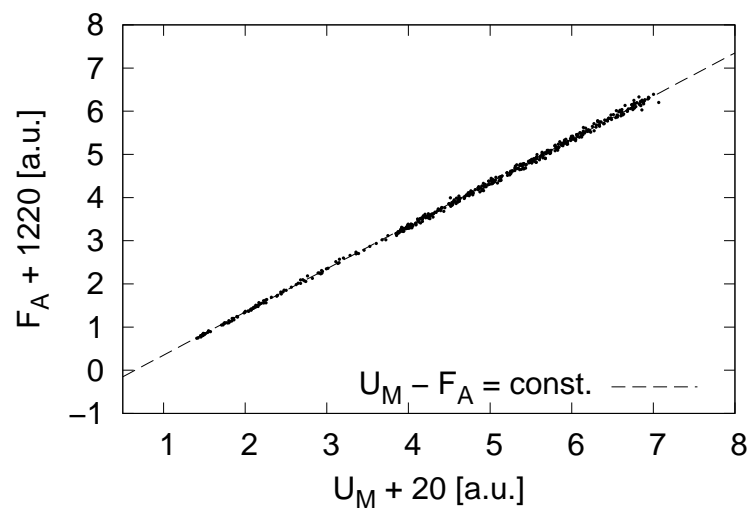

Fig. 2. Model inter-atomic potential energy $U_{M}^{2}(P=0)$ is compared with the first-principles free energy $\left(F_{A}\right)$ calculated with $64 \mathrm{Mg}$ and $64 \mathrm{O}$ atoms contained in a fcc cell. The $\Gamma$ point was sampled in the first Brillouin zone. Each dot represents a down sampled configuration in the multicanonical ensemble. 500 configurations were calculated.

first-principles calculation agree with each other very well. This means that the $U_{M}^{2}(P=0)$ model potential determined in the 64 -atom cubic cell had converged satisfactorily.

\section{Comparison between exchange-correlation potentials}

The present paper compares results using different exchange-correlation (XC) potentials. However, to compare them, the residual of thermodynamic downfolding has to be smaller than the difference due to the $\mathrm{XC}$ potentials. To confirm this, first-principles calculations using different $\mathrm{XC}$ potentials were performed on the down-sampled multicanonical simulation runs, $\mathcal{Q}^{1}(P=0)$. The XC potentials used were PBE, PBEsol, CAPZ, and PW91. This comparison on the set $\mathcal{Q}^{1}(P=0)$ has thermodynamic meaning because the set represents the thermodynamics of the system.

The results are summarized in Figs. 3 and 4. In these figures, there are groups of dots. Each dot in a group is a member in $\mathcal{Q}^{1}(P=0)$. There is a marked line immediately below each group. The marking indicates a corresponding XC potential found in the legend below the figure. Each line also determines a relation $x=y+$ const., where $x(y)$ is the vertical (horizontal) axis.

Figure 3 shows the comparison between the calculation using PBE and the calculations using the other XC potentials. The PW91 curve agrees very well with that for PBE. Because PBE was developed as a simplified version of PW91 and was expected to be close to it, this agreement is considered normal. The PBEsol curve, however, clearly has a steeper gradient than unity. This trend is even more significant for the CAPZ curve.

Figure 4 shows the comparison between $U_{M}^{2}(P=0)$ and the first-principles calculations. The dots for PBE completely follow the line with no trend. This means $U_{M}^{2}(P=0)$ was 


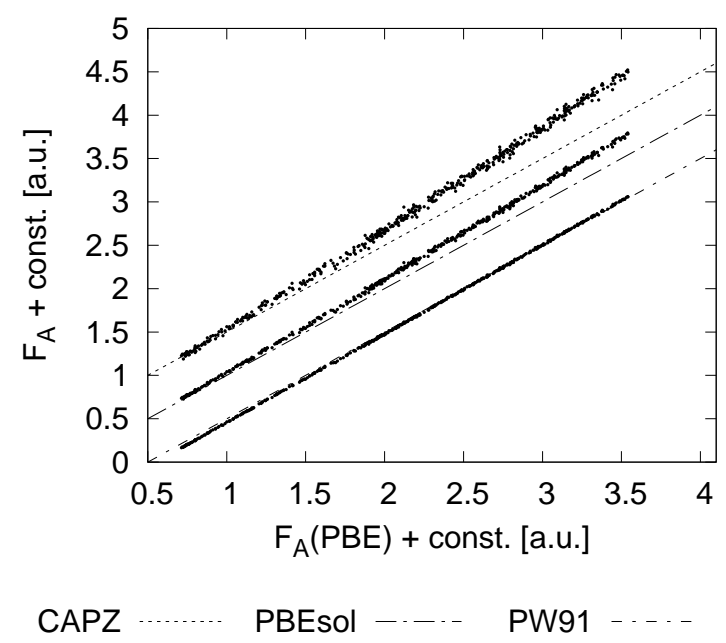

Fig. 3. Comparison of PBE XC potential with CAPZ, PBEsol and PW91 XC potentials on the multicanonical simulation run, $\mathcal{Q}^{1}(P=0)$. Each axis displays the free energy of the system.

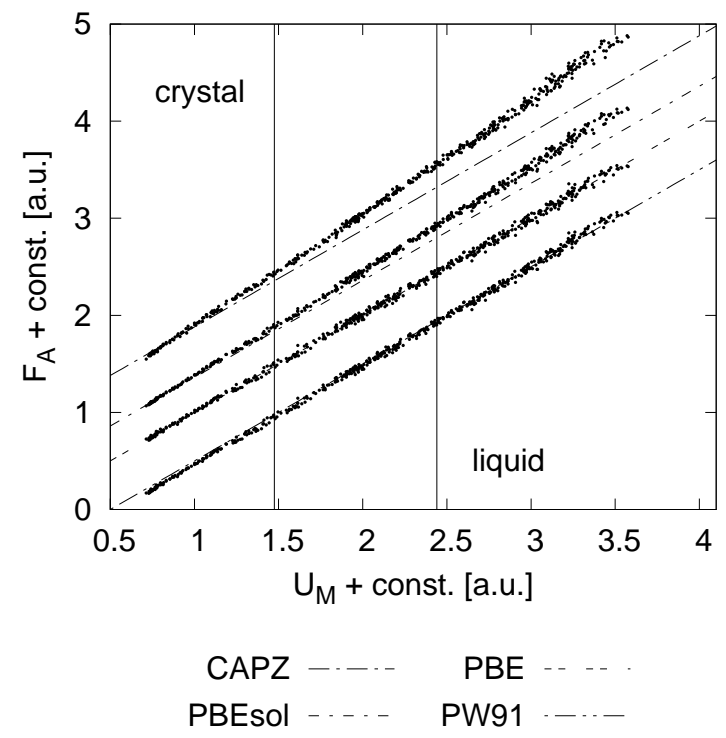

Fig. 4. Comparison of $U_{M}^{2}(P=0)$ with first-principles calculations using CAPZ, PBE, PBEsol and PW91 XC potentials on the multicanonical simulation run, $\mathcal{Q}^{1}(P=0)$. Each axis displays the free energy of the system. The left(right) vertical line shows the potential energy of crystalline(liquid) state at the melting point.

appropriately generated. The spread of dots around the line is because of the residual in downfolding. Points associated with PBEsol and CAPZ have steeper gradient than unity in this comparison as in Fig. 3. These trends are distinct compared with the spread of points. This means that the form of the present model inter-atomic potential can distinguish between PBE, PBEsol and CAPZ. The dots for PW91, however, follow the line also with no distinct 
J. Phys. Soc. Jpn.

Full PAPER

trend. This means the form of the present model potential can not distinguish any difference between PW91 and PBE if such differences existed.

In consequence, the effectiveness of the present method has been verified. Moreover, this result proves that the present approach, which falls in principle in the category of ab-initio methods, has in practice the capability of an ab-initio method.

\section{Melting at $P=0 \mathrm{GPa}$}

The result at $P=0 \mathrm{GPa}$ is summarized and compared with existing theoretical and experimental results in Table II. The table also includes the present results simulated in a 216-atom cell. The correspondence between the 64- and 216-atom cells has been discussed in $\S 6$.

From theoretical results given by Alfè, the generalized gradient approximation (GGA) produced a significantly lower melting point compared with the experimental value. In contrast, the local density approximation (LDA) used in his study produced a closer melting point. However, melting points obtained with PBE or CAPZ in the 64-atom cell rose by 400-450 K more than that with either GGA or LDA established by Alfè. A partial reason for this increase is probably because of the effect of the number of the atoms in the cell, because the present results in 216-atom cell were higher by only 200-300 K over those by Alfè. (Alfè estimated the errors to be $50 \mathrm{~K}$ and $100 \mathrm{~K}$ for LDA and GGA, respectively.)

However, even if the effect of the number of the atoms is taken into account, the present melting point with CAPZ, which is a typical LDA, was not so significantly better than that with PBE, which is a typical GGA, in contradistinction to results reported by Alfè. The present melting point with PBEsol was higher by $\sim 250 \mathrm{~K}$ than that with PBE and in 216 atom cell case was closer to the experimental value.

In addition, the present results for latent heat were rather close to recent experimental indications $\left(\mathrm{JANAF}^{13)}\right.$ and Howard $\left.{ }^{32)}\right)$. This is again in contrast to the LDA result by Alfè, which was by a wide margin larger than experimental indications. The present values of $\Delta H$ are arranged in ascending order as $\Delta H$ (experimental) $<\Delta H(\mathrm{PBE})<\Delta H(\mathrm{PBEsol})<$ $\Delta H(\mathrm{CAPZ})$. This ordering is consistent with the mean errors in atomization energy using these XC potentials. ${ }^{14,15)}$ In a related matter, Tangney and Scandolo obtained the energy difference between the liquid and crystal as a function of temperature when they studied the melting slope. ${ }^{12)}$ The difference was $\sim 79 \mathrm{~kJ} \cdot \mathrm{mol}^{-1}$ in the range of $\sim 2950 \mathrm{~K}<T<\sim 3250 \mathrm{~K}$. Although they did not give a concluding number for $T_{m}$, their result means $\Delta H \sim 79 \mathrm{~kJ} \cdot \mathrm{mol}^{-1}$. Their simulation cell contained 64 atoms and they used LDA. The difference between their result and $\Delta H(\mathrm{CAPZ})$ is understandable because they expected error of $10 \%$ and they does not seem to have included the thermal electronic excitation effect. The effect caused $6 \mathrm{~kJ} \cdot \mathrm{mol}^{-1}$ change in this study. $(\S 6)$

Possible reasons for the discrepancy in melting point between the present result with CAPZ 
Table II. Summary of results at $P=0 \mathrm{GPa}$ compared with those by existing theoretical and experimental works. $T_{m}, \Delta V, \Delta H$, and $\Delta S$ are melting point, volume change per atom during melting, latent heat, and entropy change per atom during melting, respectively. For present results, the 216-atom cell results are also shown in parentheses. (See $\S 6$ for their details.)

\begin{tabular}{|c|c|c|c|c|}
\hline & $T_{m}$ & $\Delta V$ & $\Delta H$ & $\Delta S$ \\
\hline & $\mathrm{K}$ & $\AA^{3}$ & $\mathrm{~kJ} \cdot \mathrm{mol}^{-1}$ & $k_{B}$ \\
\hline Present: PBE & $2975(2820)$ & $3.52(3.22)$ & $85(82)$ & $1.7(1.7)$ \\
\hline Present: CAPZ & $3460(3270)$ & $2.84(2.53)$ & $94(88)$ & $1.6(1.6)$ \\
\hline Present: PBEsol & $3230(3060)$ & $3.01(2.74)$ & $87(86)$ & $1.6(1.7)$ \\
\hline Alfè: GGA ${ }^{10)}$ & 2533 & & & \\
\hline Alfè: LDA ${ }^{10)}$ & 3070 & 3.08 & 112 & 2.19 \\
\hline $\mathrm{JANAF}^{13)}$ & 3100 & & 78 & 1.5 \\
\hline Howard ${ }^{32)}$ & & & 74 & \\
\hline
\end{tabular}

and that with LDA by Alfè are as follows. (1) The present approximation for thermal electronic excitations may be insufficient. (2) In the two phase simulation by Alfé, the simulation cell was fixed. ( $N V E$ simulation) This might cause an unintended shift of $T_{m}$. To study further this discrepancy in melting point, a thermodynamic integration approach with a first-principles calculation starting from the present model potential will be appropriate. The parameters of the model potentials are listed in Tables C.1, C.2, C.3, C.4, C.5, and C.6 in Appendix C. These tables report the inter-atomic potentials for both free energy and energy.

\section{Pressure dependence of melting}

The dependence on pressure of melting point, latent heat, and volume change during melting is shown in Figs. 5, 6, and 7, respectively. The numbers are also listed in Table B.1 in Appendix B.

To obtain the pressure dependence, two methods were tried. The first is to downfold the model potential at $P=0 \mathrm{GPa}$ and to use it for all other pressures. [ TD(@ 0GPa)] The second is to downfold the model potential at each pressure. [ TD ] The latter should yield a better result but the former has the advantage of a lower computational cost. The convergence of TD in terms of downfolding should be sufficient. This is because the results obtained by the first generation of downfolding, $U_{M}^{1}(P=0)$, agree well with those by the second one, $U_{M}^{2}(P=0)$ (Table B.1). This means that one generation from $U_{M}^{1}(P), P>0$, namely, $U_{M}^{2}(P), P>0$ is sufficient.

The results obtained by TD(@ $0 \mathrm{GPa})$ and TD agree rather well as can be seen in Figs. 5,6 , and 7 . In particular, the agreements are rather good for melting points and for volume changes during melting. 


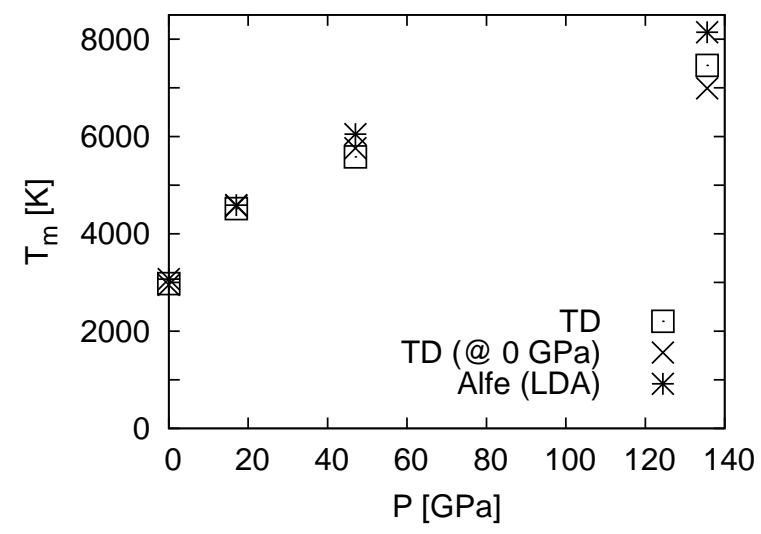

Fig. 5. Melting point as a function of pressure. TD, TD(@ 0GPa), and Alfè(LDA) are the present result by $U_{M}^{2}$, that by $U_{M}^{1}$, and the result with LDA by Alfè, ${ }^{10)}$ respectively.

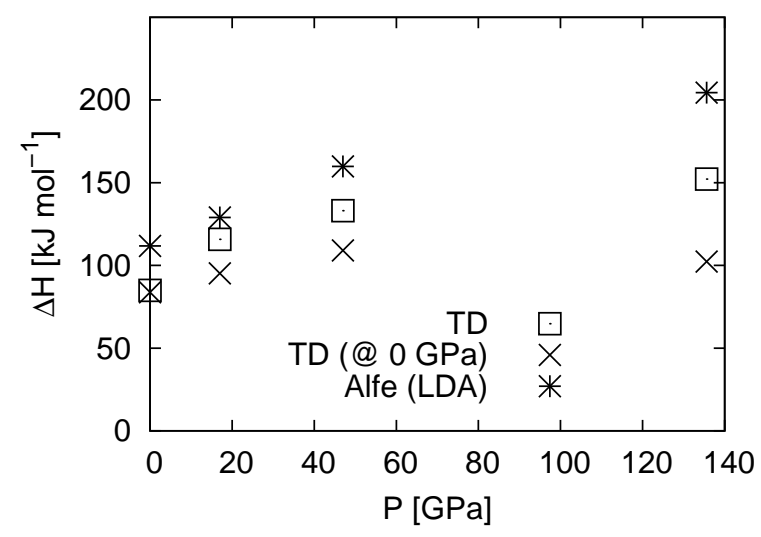

Fig. 6. Latent heat as a function of pressure. TD, TD(@ 0GPa), and Alfè(LDA) are the present result by $U_{M}^{2}$, that by $U_{M}^{1}$, and the result with LDA by Alfè, ${ }^{10)}$ respectively.

These results mean that the present approach has worked remarkably well. If the required accuracy permits, we can generate the potential at any specific pressure and use it for other nearby pressures.

Alternatively, the pressure dependence obtained here is similar to the result of Alfè. Namely, the present result also indicates a similar discrepancy between the first-principles and experimental results..$^{9,11)}$

A possible reason of this discrepancy in the pressure dependence is an $\mathrm{XC}$ potential issue. Because a claim is made that the new potential PBEsol is better for condensed materials, it is worth while to perform the present simulation with PBEsol in addition.

To obtain the result with PBEsol, one generation of downfolding starting with $U_{M}^{1}$ was performed with PBEsol. The convergence of the generated potential, $\hat{U}_{M}^{3}$, is sufficient because 


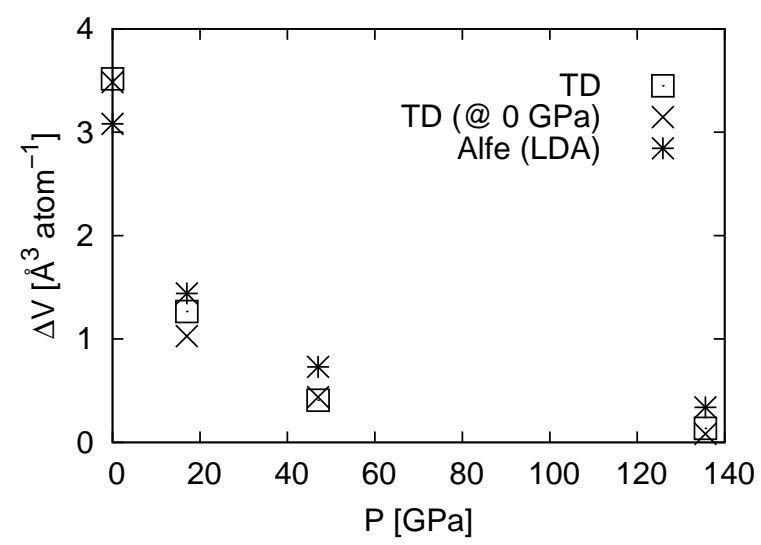

Fig. 7. Volume change during melting as a function of pressure. TD, TD(@ 0GPa), and Alfè(LDA) are the present result by $U_{M}^{2}$, that by $U_{M}^{1}$, and the result with LDA by Alfè, ${ }^{10)}$ respectively.

the results obtained with $\hat{U}_{M}^{3}(P=0)$ agree well with those with $\hat{U}_{M}^{4}(P=0)$, which represents the second generation. (Table B.1)

The obtained melting point, latent heat and volume change during melting are shown in Figs. 8, 9, and 10, respectively. As shown in these figures, the results with PBE and PBEsol $\mathrm{XC}$ potentials are quite close. In addition, these results are similar to the LDA result by Alfè. Thus, the discrepancy between theoretical and experimental works is not resolved even with PBEsol.

To study further the discrepancy arising for the electronic correlation, we should try a more accurate method such as the diffusion quantum Monte Carlo method. A reason for doing this is that the melting transition accompanies the closing of the gap in the electronic structure, and PBEsol also has a well-known gap issue like CAPZ and PBE. The present approach can be combined with such accurate methods because there is no restriction on $U_{A}$.

In addition to the direct comparison of $T_{m}$ so far, the melting slope $\left(d T_{m} / d P\right)$ at $P=0$ $\mathrm{GPa}$ is calculated using the Clausius-Clapeyron relation. The results are shown in Table III with other theoretical and experimental results. All of the theoretical results in the table were based on density functional calculations directly (ref. 10) or indirectly (ref. 12 and 34). The present results are similar to other theoretical results including the one by Tangney and Scandolo. Thus, a discrepancy similar to the one in the direct comparison of $T_{m}$ is observed in $d T_{m} / d P$ also.

Besides the theoretical aspect of the discrepancy so far discussed, we should consider the experimental aspect in addition. Recently, Adebayo et al. suggested a possible reason for the discrepancy. ${ }^{33)}$ They studied infrared absorption of $\mathrm{MgO}$ at high pressure and temperature by a MD. They found that the infrared absorption of crystalline $\mathrm{MgO}$ at $\mathrm{CO}_{2}$ laser frequencies increases substantially with both pressure and temperature. On the other hand, Zerr and 
Table III. The melting slope $d T_{m} / d P[\mathrm{~K} / \mathrm{GPa}]$ at $P=0$ GPa. PBE, CAPZ and PBEsol are the present results with the corresponding XC potentials. In the parentheses, the 216 atom results are also given (See $\S 6$ for their details). Experimental results are marked by asterisks.

\begin{tabular}{cccccccc}
\hline PBE & CAPZ & PBEsol & $\begin{array}{c}\text { Alfè } \\
\text { 10) }\end{array}$ & $\begin{array}{c}\text { Aguado and } \\
\text { Madden }^{34)}\end{array}$ & $\begin{array}{c}\text { Tangney and } \\
\text { Scandolo }^{12}\end{array}$ & $\begin{array}{c}\text { Zerr and } \\
\text { Boehler }^{9)}\end{array}$ & $\begin{array}{c}\text { Zhang } \\
\text { and Fei }^{11)}\end{array}$ \\
\hline 148 & 126 & 134 & 102 & 125 & $\sim 130-\sim 150$ & $36^{*}$ & $221^{*}$ \\
$(133)$ & $(113)$ & $(118)$ & & & & & \\
\hline
\end{tabular}

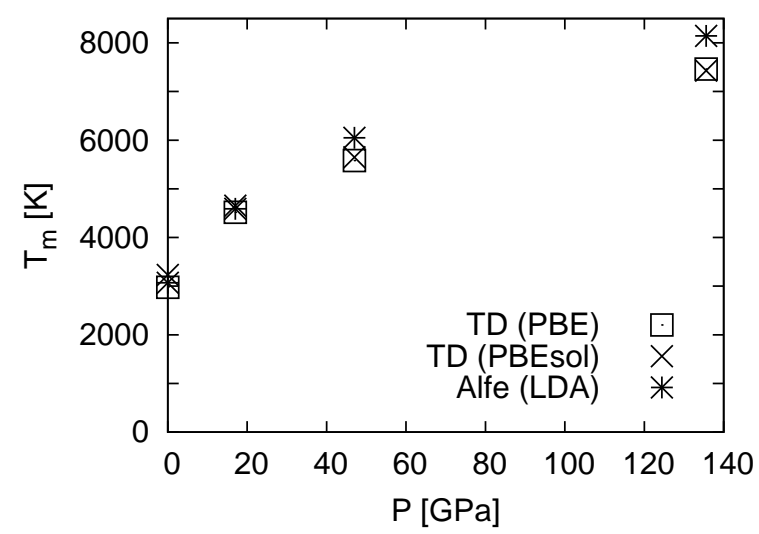

Fig. 8. Melting point as a function of pressure. TD(PBE), TD(PBEsol), and Alfè(LDA) are the present result by $U_{M}^{2}$ based on PBE, that by $\hat{U}_{M}^{3}$ based on PBEsol, and the result with LDA by Alfè, ${ }^{10)}$ respectively.

Boehler observed the abruptness of the absorption changes with laser intensity to detect the melting. However, Adebayo et al. claimed that this abruptness is not necessarily caused by the melting but can be caused by the nonlinear absorption change in several way. Although I regard this as a possible reason, I also remark that their opinion seems to have a difficulty to explain why Zerr and Boehler obtained the established melting temperature under $P=0$ GPa. If the nonlinear mechanism is absent for this pressure, we expect some singular point in the melting curve because of the possible mechanism change. The melting curve given by Zerr and Boehler, however, does not have such a singular point.

\section{Conclusion}

In conclusion, melting of $\mathrm{MgO}$ has been successfully simulated with a combination of a multicanonical ensemble method and first-principles calculation. To take into account thermal excitations of electrons within the framework of the multicanonical simulation, an approximation to incorporate the effect into a model inter-atomic potential has been introduced. The present study used a rather simple two-body model inter-atomic potential in thermodynamic 


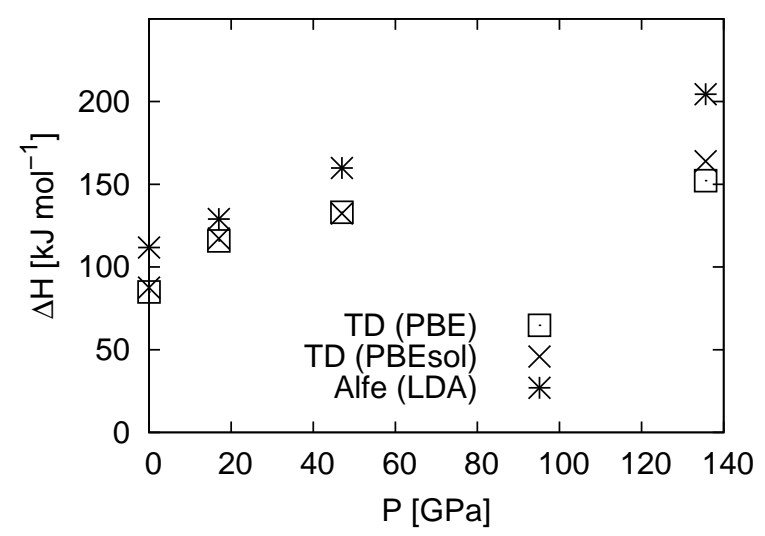

Fig. 9. Latent heat as a function of pressure. TD(PBE), TD(PBEsol), and Alfè(LDA) are the present result by $U_{M}^{2}$ based on PBE, that by $\hat{U}_{M}^{3}$ based on PBEsol, and the result with LDA by the Alfè, ${ }^{10)}$ respectively.

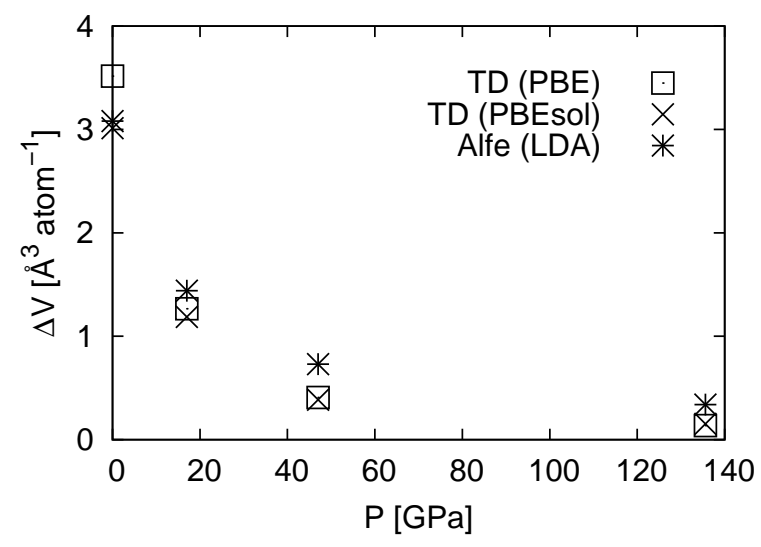

Fig. 10. Volume change during melting as a function of pressure. TD(PBE), TD(PBEsol), and Alfè(LDA) are the present result by $U_{M}^{2}$ based on PBE, that by $\hat{U}_{M}^{3}$ based on PBEsol, and the result with LDA by Alfè, ${ }^{10)}$ respectively.

downfolding. Significantly, the Ewald term was not contained in the potential. Nevertheless, thermodynamic downfolding could distinguish differences due to the $\mathrm{XC}$ potentials used in a first-principles calculations.

Under $0 \mathrm{GPa}$, the present method was performed separately with PBE, PBEsol, and CAPZ $\mathrm{XC}$ potentials. Between them, PBEsol seems to give a melting point closest to experimental values. The present values for latent heat using these $\mathrm{XC}$ potentials were rather close to recent experimental results. This is in contrast to the LDA result of Alfè, which was far larger than those suggested experimentally. Of the $\mathrm{XC}$ potentials used, $\mathrm{PBE}$ values came closest to experiments. This was to be expected from the mean errors in atomization energy for these $\mathrm{XC}$ potentials. 
To obtain the pressure dependence, two methods were tried. The first was to downfold the model potential at $P=0 \mathrm{GPa}$ and to use results for all other pressures. The second was to downfold the model potential at each pressure. Results showed that these two methods agreed well one with the other. This suggests that the present approach worked remarkably well.

The obtained pressure dependence was similar to the previous study by Alfè. PBEsol, which is a revised parameterization of $\mathrm{PBE}$ and claims better performance for condensed systems, did not change this dependence. Therefore, the discrepancy between first-principles and experimental studies unfortunately still remains in the melting curve of $\mathrm{MgO}$. The alternative comparison of the melting slope at $P=0 \mathrm{GPa}$ also shows a similar discrepancy, which was also claimed by Tangney and Scandolo.

\section{Acknowledgments}

This work is partially supported by a Grant-in-Aid for Young Scientists (B) of the Ministry of Education, Culture, Sports, Science, and Technology (MEXT), Japan, by a Grant-in-Aid for Scientific Research in Priority Areas "Development of New Quantum Simulators and Quantum Design" (No.17064004) of the MEXT, Japan, and by the Next Generation Super Computing Project, Nanoscience Program, MEXT, Japan. The computation in this work had been done using the facilities of the Supercomputer Center, Institute for Solid State Physics, University of Tokyo, the facilities of Supercomputing Division, Information Technology Center, The University of Tokyo, and a facility of Institute for Molecular Science, National Institute of Natural Science, Japan.

\section{Appendix A: Technical improvements for larger simulation cells}

For larger simulation cells, two additional technical improvements were introduced.

First, a minor control affecting the Wang-Landau factor was set up.

In large simulation cells, nearly perfect crystalline orderings become possible. These are characterized by the reciprocal lattice vectors whose lengths are nearly the same as those for perfect crystalline ordering. These nearly perfect structures are irrelevant in the re-weighting to calculate physical quantities because they are observed in the small entropy area of (energy, order parameter) space in current system sizes. However, these become traps during simulations.

These traps can be avoided in the learning process of the Wang-Landau algorithm by imposing a penalty on the Wang-Landau factor when these are accessed. Specifically, the factor was multiplied by $A_{P}=32$ when

$$
\frac{1}{N_{A}^{p}} \sqrt{\frac{1}{\left|\mathcal{G}_{\text {avd }}\right|} \sum_{\mathbf{G} \in \mathcal{G}_{\text {avd }}}|s(\mathbf{G})|^{2 p}}
$$

becomes larger than a specified threshold. The number of reciprocal vectors $\mathcal{G}_{\text {avd }}$ needed to invoke this avoidance was 96 for the 128-atom fcc cell and 144 for 216-atom cubic cell, 
J. Phys. Soc. Jpn.

Full PAPER

respectively. The exponent $p=3$ sharpens the discrimination of the quasi-order. The threshold was 0.07 for the 128 -atom fcc cell and 0.02 for the 216 -atom cubic cell. In production runs, this "avoidance" is disabled so as to keep physical quantities.

For production runs, a related improvement is also available. In the learning process, we record a histogram for the application of this penalty. The area in (energy, order parameter) space where this histogram is above a suitable threshold can be regarded as the trapping area. Exploiting this fact, we define an appropriate function that is positive on the trapping area and zero otherwise. We can make the production run avoid the area by adding this function to the multicanonical weight. An example of the function is the logarithm of the histogram itself. When the height of the function is $3 k_{B}$, the probability of observing the system in the area is reduced to $1 / \exp (3)$.

Second, for the 216-atom cubic cell, the definition of the scaled order parameter changes to

$$
O=3\left(\frac{O_{n s}}{O_{\max }}\right)^{\alpha}-\frac{3}{2}
$$

where $O_{\max }$ and $\alpha$ are 108 and $\log (2 / 3) / \log (1 / 2)$, respectively. The number of shortest reciprocal lattice vectors was 32 for this cell. This number is large because the atomic arrangement of perfect crystalline order is not unique in this cell. Nevertheless, the number of simultaneously active reciprocal vectors was 8 and consequently normalization by the set size $|\mathcal{G}|$ in the definition of $O_{n s}$ was decreased to 8 for this case. Also the effective range of $O$ was from $-3 / 2$ to $3 / 2$ by this definition.

\section{Appendix B: Details of the results}

In Table B.1, details are listed of the results from $U_{M}^{1}, U_{M}^{2}, \hat{U}_{M}^{3}, \hat{U}_{M}^{4}$ and $\tilde{U}_{M}^{4} \cdot U_{M}^{1}$ and $U_{M}^{2}$ used PBE, $\hat{U}_{M}^{3}$ and $\hat{U}_{M}^{4}$ used PBEsol and $\tilde{U}_{M}^{4}$ used CAPZ.

\section{Appendix C: Downfolded model inter-atomic potentials}

The downfolded parameters for the model inter-atomic potentials are listed in Tables $\mathrm{C} \cdot 1, \mathrm{C} \cdot 2, \mathrm{C} \cdot 3, \mathrm{C} \cdot 4, \mathrm{C} \cdot 5, \mathrm{C} \cdot 6$, and C.7. In these tables, $U$ and $E$ are the free energy and its associated energy inter-atomic potentials, respectively. Their functional form is presented in the main text (eq. 6). Both potentials had this same functional form. The value of $f_{0}$ was $8.4333463 \times 10^{-4}$. The parameters not given in the tables were set to zero.

\section{Appendix D: Computational costs}

It is difficult to compare computational costs between different theoretical works, because the details in algorithms, program codes and hardware become important. In addition, the present study used a variety of computers. Nevertheless, to give some idea of the required computational costs for the present study, costs for one iteration of downfolding and the following MOMT simulation is presented here. 
Table B.1. Summary of the results for a 64 atom cubic cell. $P, T_{m}, V_{x}, V_{l}, \Delta V, \Delta H$, and $\Delta S$ are pressure, melting point, volume per atom for crystalline state at $T_{m}$, volume per atom for liquid state at $T_{m}$, volume change per atom during melting, latent heat, and entropy change per atom during melting, respectively. The far left column displays the model inter-atomic potential used while the far right column displays the XC potential used.

\begin{tabular}{|c|c|c|c|c|c|c|c|c|}
\hline & $\begin{array}{c}P \\
\mathrm{GPa}\end{array}$ & $\begin{array}{c}T_{m} \\
\mathrm{~K} \\
\end{array}$ & $\begin{array}{l}V_{x} \\
\AA^{3} \\
\end{array}$ & $\begin{array}{l}V_{l} \\
\AA^{3} \\
\end{array}$ & $\begin{array}{r}\Delta V \\
\AA^{3} \\
\end{array}$ & $\begin{array}{c}\Delta H \\
\mathrm{~kJ} \cdot \mathrm{mol}^{-1}\end{array}$ & $\begin{array}{l}\Delta S \\
k_{B}\end{array}$ & \\
\hline$U_{M}^{1}(P=0)$ & 0 & 2950 & 10.94 & 14.42 & 3.49 & 84 & 1.7 & PBE \\
\hline$U_{M}^{1}(P=0)$ & 17 & 4575 & 10.03 & 11.05 & 1.03 & 95 & 1.3 & PBE \\
\hline$U_{M}^{1}(P=0)$ & 47 & 5760 & 8.74 & 9.18 & 0.44 & 109 & 1.1 & PBE \\
\hline$U_{M}^{1}(P=0)$ & 135.6 & 6990 & 6.94 & 7.03 & 0.08 & 102 & 0.9 & PBE \\
\hline$U_{M}^{2}(P=0)$ & 0 & 2975 & 10.93 & 14.44 & 3.52 & 85 & 1.7 & PBE \\
\hline$U_{M}^{2}(P=17)$ & 17 & 4519 & 9.98 & 11.25 & 1.27 & 116 & 1.5 & PBE \\
\hline$U_{M}^{2}(P=47)$ & 47 & 5580 & 8.84 & 9.24 & 0.41 & 133 & 1.4 & PBE \\
\hline$U_{M}^{2}(P=135.6)$ & 135.6 & 7460 & 7.12 & 7.25 & 0.14 & 152 & 1.2 & PBE \\
\hline$\hat{U}_{M}^{3}(P=0)$ & 0 & 3230 & 10.66 & 13.67 & 3.01 & 87 & 1.6 & PBEsol \\
\hline$\hat{U}_{M}^{3}(P=17)$ & 17 & 4655 & 9.77 & 10.96 & 1.19 & 117 & 1.5 & PBEsol \\
\hline$\hat{U}_{M}^{3}(P=47)$ & 47 & 5650 & 8.67 & 9.05 & 0.39 & 132 & 1.4 & PBEsol \\
\hline$\hat{U}_{M}^{3}(P=135.6)$ & 135.6 & 7430 & 6.99 & 7.15 & 0.15 & 164 & 1.3 & PBEsol \\
\hline$\hat{U}_{M}^{4}(P=0)$ & 0 & 3170 & 10.69 & 14.00 & 3.31 & 85 & 1.6 & PBEsol \\
\hline$\tilde{U}_{M}^{4}(P=0)$ & 0 & 3460 & 10.43 & 13.26 & 2.84 & 93.9 & 1.6 & CAPZ \\
\hline
\end{tabular}

Table C.1. Downfolded parameters for the $U_{M}^{1}(P=0)$ and its associated $E_{M}^{1}(P=0)$ potentials. All non-dimensionless values are in atomic units.

\begin{tabular}{cccccccccc}
\hline & $a_{\mathrm{Mg}, \mathrm{Mg}}$ & $a_{\mathrm{Mg}, \mathrm{O}}$ & $a_{\mathrm{O}, \mathrm{O}}$ & $b_{\mathrm{Mg}, \mathrm{Mg}}$ & $b_{\mathrm{Mg}, \mathrm{O}}$ & $b_{\mathrm{O}, \mathrm{O}}$ & $d_{\mathrm{Mg}, \mathrm{O}}$ & $\beta_{\mathrm{Mg}, \mathrm{O}}$ & $r_{\mathrm{Mg}, \mathrm{O}}^{0}$ \\
\hline$U$ & 9.037007 & 8.922508 & 9.139069 & 1.030136 & 1.019860 & 1.050079 & 0.440839 & 0.938921 & 2.515958 \\
$E$ & 9.521568 & 11.222058 & 9.984526 & 1.120906 & 1.096115 & 1.196248 & 5.604914 & 0.894860 & 1.060691 \\
\hline
\end{tabular}

Table C.2. Downfolded parameters for the $U_{M}^{2}(P)$ potentials. $P$ is in GPa. All non-dimensionless values are in atomic unit.

\begin{tabular}{lccccccccc}
\hline \multicolumn{1}{c}{$P$} & $a_{\mathrm{Mg}, \mathrm{Mg}}$ & $a_{\mathrm{Mg}, \mathrm{O}}$ & $a_{\mathrm{O}, \mathrm{O}}$ & $b_{\mathrm{Mg}, \mathrm{Mg}}$ & $b_{\mathrm{Mg}, \mathrm{O}}$ & $b_{\mathrm{O}, \mathrm{O}}$ & $\beta_{\mathrm{Mg}, \mathrm{O}}$ & $d_{\mathrm{Mg}, \mathrm{O}}$ & $r_{\mathrm{Mg}, \mathrm{O}}^{0}$ \\
\hline 0 & 9.511351 & 10.285953 & 9.227619 & 1.147506 & 1.032505 & 1.064371 & 2.457641 & 0.941071 & 1.571412 \\
17 & 7.805613 & 10.839585 & 7.749410 & 0.758634 & 1.249861 & 0.783224 & 0.757560 & 0.879671 & 2.328365 \\
47 & 8.508896 & 9.993460 & 8.096387 & 0.914784 & 1.121834 & 0.867463 & 0.682049 & 0.915604 & 2.309614 \\
135.6 & 8.991686 & 9.402277 & 8.207214 & 0.990086 & 1.007392 & 0.873787 & 0.847770 & 0.967281 & 2.113917 \\
\hline
\end{tabular}


Table C.3. Downfolded parameters for the $E_{M}^{2}(P)$ potentials associated with $U_{M}^{2}(P) . P$ is in GPa.

All non-dimensionless values are in atomic unit.

\begin{tabular}{llllcccccc}
\hline \multicolumn{1}{c}{$P$} & \multicolumn{1}{c}{$a_{\mathrm{Mg}, \mathrm{Mg}}$} & \multicolumn{1}{c}{$a_{\mathrm{Mg}, \mathrm{O}}$} & $a_{\mathrm{O}, \mathrm{O}}$ & $b_{\mathrm{Mg}, \mathrm{Mg}}$ & $b_{\mathrm{Mg}, \mathrm{O}}$ & $b_{\mathrm{O}, \mathrm{O}}$ & $d_{\mathrm{Mg}, \mathrm{O}}$ & $\beta_{\mathrm{Mg}, \mathrm{O}}$ & $r_{\mathrm{Mg}, \mathrm{O}}^{0}$ \\
\hline 0 & 10.057433 & 10.958293 & 10.093550 & 1.238631 & 1.117503 & 1.220149 & 3.077068 & 0.868479 & 1.364818 \\
17 & 8.586335 & 9.654085 & 8.928195 & 0.904799 & 0.904578 & 0.980405 & 3.180478 & 1.065285 & 1.609773 \\
47 & 8.802583 & 9.027273 & 8.648471 & 0.943127 & 0.832501 & 0.910779 & 2.431115 & 1.128023 & 1.785129 \\
135.6 & 8.834105 & 9.124699 & 8.447506 & 0.940447 & 0.924673 & 0.857453 & 0.997715 & 1.032836 & 2.236116 \\
\hline
\end{tabular}

Table C.4. Downfolded parameters for the $\hat{U}_{M}^{3}(P)$ potentials based on PBEsol. $P$ is in GPa. All non-dimensionless values are in atomic unit.

\begin{tabular}{lccccccccc}
\hline \multicolumn{1}{c}{$P$} & $a_{\mathrm{Mg}, \mathrm{Mg}}$ & $a_{\mathrm{Mg}, \mathrm{O}}$ & $a_{\mathrm{O}, \mathrm{O}}$ & $b_{\mathrm{Mg}, \mathrm{Mg}}$ & $b_{\mathrm{Mg}, \mathrm{O}}$ & $b_{\mathrm{O}, \mathrm{O}}$ & $d_{\mathrm{Mg}, \mathrm{O}}$ & $\beta_{\mathrm{Mg}, \mathrm{O}}$ & $r_{\mathrm{Mg}, \mathrm{O}}^{0}$ \\
\hline 0 & 9.616249 & 10.416043 & 9.381960 & 1.169388 & 1.056588 & 1.104599 & 2.464106 & 0.918641 & 1.526871 \\
17 & 7.809186 & 10.902950 & 7.719037 & 0.760681 & 1.237988 & 0.783028 & 0.878400 & 0.879261 & 2.240094 \\
47 & 8.479070 & 9.991900 & 8.049807 & 0.910601 & 1.120429 & 0.864588 & 0.692440 & 0.917065 & 2.299699 \\
135.6 & 8.946077 & 9.367895 & 8.160640 & 0.984307 & 1.001234 & 0.870410 & 0.852599 & 0.974624 & 2.113341 \\
\hline
\end{tabular}

Table C.5. Downfolded parameters for the $\hat{E}_{M}^{3}(P)$ potentials associated with $\hat{U}_{M}^{3}(P)$. (based on PBEsol) $P$ is in GPa. All non-dimensionless values are in atomic unit.

\begin{tabular}{llllcccccc}
\hline \multicolumn{1}{c}{$P$} & \multicolumn{1}{c}{$a_{\mathrm{Mg}, \mathrm{Mg}}$} & \multicolumn{1}{c}{$a_{\mathrm{Mg}, \mathrm{O}}$} & \multicolumn{1}{c}{$a_{\mathrm{O}, \mathrm{O}}$} & $b_{\mathrm{Mg}, \mathrm{Mg}}$ & $b_{\mathrm{Mg}, \mathrm{O}}$ & $b_{\mathrm{O}, \mathrm{O}}$ & $d_{\mathrm{Mg}, \mathrm{O}}$ & $\beta_{\mathrm{Mg}, \mathrm{O}}$ & $r_{\mathrm{Mg}, \mathrm{O}}^{0}$ \\
\hline 0 & 10.173488 & 11.031455 & 10.223373 & 1.262270 & 1.142935 & 1.252087 & 2.803954 & 0.848087 & 1.375338 \\
17 & 8.583008 & 9.650199 & 8.930708 & 0.905555 & 0.904484 & 0.986814 & 3.202733 & 1.064061 & 1.600048 \\
47 & 8.763038 & 8.978098 & 8.598636 & 0.936597 & 0.828123 & 0.905677 & 2.346935 & 1.132265 & 1.802724 \\
135.6 & 8.779660 & 9.148105 & 8.390658 & 0.932058 & 0.931337 & 0.850587 & 0.969167 & 1.033046 & 2.259133 \\
\hline
\end{tabular}

Table C.6. Downfolded parameters for the $\hat{U}_{M}^{4}(P=0)$ and its associated $\hat{E}_{M}^{4}(P=0)$ potentials. These were based on PBEsol. All non-dimensionless values are in atomic unit.

\begin{tabular}{cccccccccc}
\hline & $a_{\mathrm{Mg}, \mathrm{Mg}}$ & $a_{\mathrm{Mg}, \mathrm{O}}$ & $a_{\mathrm{O}, \mathrm{O}}$ & $b_{\mathrm{Mg}, \mathrm{Mg}}$ & $b_{\mathrm{Mg}, \mathrm{O}}$ & $b_{\mathrm{O}, \mathrm{O}}$ & $d_{\mathrm{Mg}, \mathrm{O}}$ & $\beta_{\mathrm{Mg}, \mathrm{O}}$ & $r_{\mathrm{Mg}, \mathrm{O}}^{0}$ \\
\hline$U$ & 8.831134 & 10.237103 & 8.777481 & 0.979987 & 1.019958 & 0.974609 & 2.384864 & 0.970088 & 1.663677 \\
$E$ & 8.831114 & 10.431142 & 8.779725 & 0.979918 & 1.015092 & 0.975064 & 3.449138 & 0.975032 & 1.474028 \\
\hline
\end{tabular}

Table C.7. Downfolded parameters for the $\tilde{U}_{M}^{4}(P=0)$ and its associated $\tilde{E}_{M}^{4}(P=0)$ potentials. These were based on CAPZ. All non-dimensionless values are in atomic unit.

\begin{tabular}{cccccccccc}
\hline & $a_{\mathrm{Mg}, \mathrm{Mg}}$ & $a_{\mathrm{Mg}, \mathrm{O}}$ & $a_{\mathrm{O}, \mathrm{O}}$ & $b_{\mathrm{Mg}, \mathrm{Mg}}$ & $b_{\mathrm{Mg}, \mathrm{O}}$ & $b_{\mathrm{O}, \mathrm{O}}$ & $d_{\mathrm{Mg}, \mathrm{O}}$ & $\beta_{\mathrm{Mg}, \mathrm{O}}$ & $r_{\mathrm{Mg}, \mathrm{O}}^{0}$ \\
\hline$U$ & 9.177385 & 10.329528 & 8.952782 & 1.052954 & 1.042900 & 1.021168 & 2.428146 & 0.939454 & 1.563449 \\
$E$ & 8.984235 & 9.8872164 & 8.922903 & 1.008925 & 0.949289 & 0.985487 & 3.216059 & 1.014374 & 1.489500 \\
\hline
\end{tabular}


In total 605 CPU hours on a SGI Altix 3700Bx2 in the Institute for Solid State Physics (ISSP) was needed to perform the first-principles calculation of thermodynamic downfolding in S2 iteration at $P=47 \mathrm{GPa}$. By parallelizing the computation, the wall-clock time for the calculation was 5 hours. The following $M O M T$ simulation in S3 iteration was performed by a node of Hitachi SR11000/J1 in ISSP. To obtain a converged $\tilde{W}, 20$ node hours was used. The production run consumed 10 node hours.

The wall clock time for the MOMT simulation is larger than that for downfolding. However, this may be because TAPP is far more developed compared with the program performing the $M O M T$ simulation. 


\section{References}

1) J. R. Morris, C. Z. Wang, K. M. Ho, and C. T. Chan: Phys. Rev. B 49 (1994) 3109-3115.

2) D. Frenkel and B. Smit: Understanding Molecular Simulation, second ed. (Academic Press, San Diego, 2002) second ed. Chapter 7.

3) M. Watanabe and W. P. Reinhardt: Phys. Rev. Lett. 65 (1990) 3301-3304.

4) O. Sugino and R. Car: Phys. Rev. Lett. 74 (1995) 1823-1826.

5) Y. Yoshimoto: J. Chem. Phys. 125 (2006) 184103.

6) B. A. Berg and T. Neuhaus: Phys. Lett. B 267 (1991) 249.

7) B. A. Berg and T. Neuhaus: Phys. Rev. Lett. 68 (1992) 9-12.

8) J. Lee: Phys. Rev. Lett. 71 (1993) 211-214.

9) A. Zerr and R. Boehler: Nature 371 (1994) 506-508.

10) D. Alfè: Phys. Rev. Lett. 94 (2005) 235701.

11) L. Zhang and Y. Fei: Geophys. Res. Lett. 35 (2008) L13302.

12) P. Tangney and S. Scandolo: J. Chem. Phys. 131 (2009) 124510.

13) M. W. Chase, Jr., C. A. Davies, J. R. Downey, Jr., D. J. Frurip, R. A. McDonald, and A. N. Syverud: JANAF Thermochemical Tables Third Edition Part II, Cr-Zr Vol. 14 of J. Phys. Chem. Ref. Data (American Chemical Society, New York, 1985) third ed.

14) J. P. Perdew, A. Ruzsinszky, G. I. Csonka, O. A. Vydrov, G. E. Scuseria, L. A. Constantin, X. Zhou, and K. Burke: Phys. Rev. Lett. 100 (2008) 136406.

15) J. P. Perdew, A. Ruzsinszky, G. I. Csonka, O. A. Vydrov, G. E. Scuseria, L. A. Constantin, X. Zhou, and K. Burke: Phys. Rev. Lett. 102 (2009) 039902.

16) F. Wang and D. P. Landau: Phys. Rev. Lett. 86 (2001) 2050-2053.

17) A. M. Ferrenberg and R. H. Swendsen: Phys. Rev. Lett. 61 (1988) 2635-2638, ibid. 631658 (1989).

18) F. Wang and D. P. Landau: Phys. Rev. E 64 (2001) 056101.

19) J. Higo, N. Nakajima, H. Shirai, A. Kidera, and H. Nakamura: J. Compt. Chem. 18 (1997) 20862092.

20) J. Yamauchi, M. Tsukada, S. Watanabe, and O. Sugino: Phys. Rev. B 54 (1996) 5586-5603.

21) J. P. Perdew, K. Burke, and M. Ernzerhof: Phys. Rev. Lett. 77 (1996) 3865-3868.

22) J. P. Perdew, K. Burke, and M. Ernzerhof: Phys. Rev. Lett. 78 (1997) 1396.

23) J. P. Perdew and A. Zunger: Phys. Rev. B 23 (1981) 5048.

24) D. M. Ceperley and B. J. Alder: Phys. Rev. Lett. 45 (1980) 566.

25) J. P. Perdew, J. A. Chevary, S. H. Vosko, K. A. Jackson, M. R. Pederson, D. J. Singh, and C. Fiolhais: Phys. Rev. B 46 (1992) 6671-6687.

26) M. Bernasconi, G. L. Chiarotti, P. Focher, S. Scandolo, E. Tosatti, and M. Parrinello: J. Phys. Chem. Solids 56 (1995) 501-505.

27) U. H. Hansmann, Y. Okamoto, and F. Eisenmenger: Chem. Phys. Lett. 259 (1996) 321-330.

28) N. Nakajima, H. Nakamura, and A. Kidera: J. Phys. Chem. B 101 (1997) 817-824.

29) H. Okumura and Y. Okamoto: Chem. Phys. Lett. 383 (2004) 391-396, ibid. 391, 248 (2004).

30) H. A. Stern: J. Comput. Chem. 25 (2004) 749-761.

31) K. Kawamura: Molecular Dynamics Simulations, ed. F. Yonezawa (Springer, Berlin, 1990) p. 88.

32) R. A. Howald: CALPHAD 16 (1992) 25-32.

33) G. A. Adebayo, Y. Liang, C. R. Miranda, and S. Scandolo: J. Chem. Phys 131 (2009) 014506. 
34) A. Aguado and P. A. Madden: Phys. Rev. Lett. 94 (2005) 068501. 